\title{
Rotavirus-Associated Necrotizing Enterocolitis After Cardiac Catheterization in Infants
}

\author{
JON N. MELIONES, M.D., THOMAS C. SHOPE, M.D., AMNON ROSENTHAL, M.D., and \\ JOHN M. PALMISANO, R.R.T. \\ From the Department of Pediatrics, C.S. Mott Children's Hospital, University of Michigan Medical Center, Ann Arbor, \\ Michigan
}

\begin{abstract}
Necrotizing enterocolitis is common in preterm and low birth weight infants but rare beyond the neonatal period. Various infectious agents including bacterial and viral organisms have been implicated in its pathogenesis. Rotavirus has recently been shown to be associated with necrotizing enterocolitis. In this report we describe two infants with congenital cardiac disease who developed fulminating necrotizing enterocolitis after cardiac catheterization. Both infants were beyond the newborn period and developed enterocolitis during an epidemic of rotaviral infection. We postulate a relationship between rotavirus infection and the development of necrotizing enterocolitis in older infants with congenital heart disease after cardiac catheterization. (J Interven Cardiol 1991:4:2)
\end{abstract}

\section{Introduction}

Necrotizing enterocolitis is a common and frequently catastrophic gastrointestinal condition primarily noted in preterm and low birth weight neonates. The disease is characterized by abdominal distention, occult or gross blood in stools, pneumatosis intestinalis, and other clinical and laboratory findings of sepsis. ${ }^{1}$ Necrotizing enterocolitis occurs in primarily endemic form with periodic epidemics. ${ }^{2}$ Specific etiology has not been determined, yet numerous bacterial and viral agents have been proposed as risk factors. $^{3}$

In this report we describe two infants with congenital heart disease who developed fulminating enterocolitis after cardiac catheterization. These cases occurred during an epidemic of rotavirus infection at our institution. From this experience

Address for Reprints: Jon N. Meliones, M.D., F1126, C.S. Mott Children's Hospital, University of Michigan Medical Center, Ann Arbor, MI 48109-0204. Fax: 313-936-9470.

Submitted for publication September 13, 1990; accepted with revisions February 6, 1991; revisions returned February 12 , 1991. we postulate that there is an association between rotavirus infection and the subsequent development of necrotizing enterocolitis in infants with congenital heart disease.

\section{Methods}

The clinical diagnosis of necrotizing enterocolitis was made when the infant's symptoms and signs fulfilled the modified staging criteria of Bell. ${ }^{1}$ A constellation of gastrointestinal symptoms and radiographic findings were present. $\mathrm{Ab}$ dominal symptoms included distention, tenderness, feeding intolerance, bilious nasogastric drainage, and diarrhea. Stools were consistently heme positive. The radiographic findings consisted of pneumatosis intestinalis and/or portal or peritoneal air.

Testing for rotavirus was performed using the quantitative enzyme immunoassay "Pathfinder," a commercially available monoclonal antibody-based immunoassay. ${ }^{4}$ The technique has been shown to be as sensitive and specific as electron microscopy identification of virus particles. 
MELIONES, ET AL.

Table 1. Patients at Risk During the 5-Month Period

\begin{tabular}{|c|c|c|c|}
\hline & $\begin{array}{l}\text { All Patients } \\
\text { Tested for } \\
\text { Rotavirus }\end{array}$ & $\begin{array}{c}\text { Patients Age } \\
<1 \text { Year } \\
\text { Who } \\
\text { Underwent } \\
\text { Cardiac } \\
\text { Cath }\end{array}$ & $\begin{array}{c}\text { Patients Age } \\
<1 \text { Year } \\
\text { Who } \\
\text { Underwent } \\
\text { Cardiac } \\
\text { Surgery }\end{array}$ \\
\hline Total patients & 270 & 35 & 46 \\
\hline $\begin{array}{l}\text { Patients positive } \\
\text { for rotavirus }\end{array}$ & 137 & 4 & 3 \\
\hline $\begin{array}{l}\text { Patients who } \\
\text { developed } \\
\text { NEC }\end{array}$ & $2^{*}$ & $2^{*}$ & $2^{* *}$ \\
\hline
\end{tabular}

* the two patients presented in this paper; ${ }^{* *}$ neither patient was tested for rotavirus but developed necrotizing enterocolitis after surgery; Cath $=$ catheterization; NEC $=$ necrotizing enterocolitis.

\section{Results}

From February through June of 1988,270 inpatients at the C.S. Mott Children's Hospital had stool samples sent for rotavirus testing (Table 1). Of these, 137 samples $(51 \%)$ were positive. Of the 137 positive samples, $70(52 \%)$ were in infants under 1 year in age. During the study period, 35 patients $<1$ year of age underwent cardiac catheterization. Of these 35 infants, five were tested for rotavirus. In one the test was negative while in four it was positive. Two of these four developed enterocolitis and are recorded as case 1 and case 2. During this same 5-month period, 46 patients under 1 year of age underwent cardiac surgery. None were tested for rotavirus in the immediate perioperative period. Three patients developed gastroenteritis more than 2 weeks after surgery and subsequently were shown to be positive for rotavirus. None of these had necrotizing enterocolitis. All recovered uneventfully. Two postsurgical patients developed enterocolitis but stools were not tested for rotavirus during those acute episodes.

\section{Case 1}

L.D., a 6-month-old female infant with a ventricular septal defect, patent ductus arteriosus and congestive heart failure, underwent cardiac catheterization. There was evidence for a nonrestric- tive ventricular septal defect and large patent ductus arteriosus resulting in a large left-to-right shunt (pulmonary blood flow three times systemic blood flow). There was pulmonary artery hypertension with the pulmonary artery pressure equaling systemic pressure. Pulmonary vascular resistance was elevated at 6 woods units which deceased to 4-woods units on $100 \%$ oxygen. Left ventricular systolic and diastolic function were normal. During the study the infant received a total of $5 \mathrm{~mL} / \mathrm{kg}$ of Iohexol (Omnipaque; Winthrop Pharmaceuticals, New York, NY, USA), a nonionic contrast agent. No complications were noted during or immediately after the procedure. Twenty-four hours after the procedure she developed diarrhea, and 48 hours later developed abdominal distention and bloody stools. An abdominal roentgenogram disclosed pneumatosis intestinalis of the transverse and descending colon. A stool sample sent during the episode of bloody stools was positive for rotavirus. The infant was treated with antibiotics for 14 days and recovered fully.

\section{Case 2}

J.S, a previously healthy 35 -day-old male, was admitted in congestive failure. The infant's birth weight was $4.5 \mathrm{~kg}$. A clinical diagnosis of critical aortic stenosis was made and the infant underwent cardiac catheterization for possible balloon valvuloplasty. During catheterization a left ventricular and aortic angiogram were performed. There was evidence for a bicuspid aortic valve with severe valvular stenosis with a peak systolic ejection gradient of $76 \mathrm{mmHg}$. In addition, there was left ventricular diastolic dysfunction (left ventricular end-diastolic pressure $=12 \mathrm{mmHg}$ ) and normal systolic function (left ventricular ejection fraction $=61 \%$ ). A total of $2 \mathrm{~mL} / \mathrm{kg}$ of Iohexol was administered. A 6-mm balloon valvulopasty catheter was placed in the left femoral artery and advanced to the descending aorta but could not be advanced any further. During removal of the balloon an intimal tear of the left iliac artery occurred. The infant remained stable but 12 hours after catheterization he developed a distended abdomen with bloody stools. Abdominal roentgenograms confirmed the presence of 


\section{NECROTIZING ENTEROCOLITIS AFTER INVASIVE CARDIAC PROCEDURES}

pneumatosis intestinalis with air in the biliary tree. Despite the administration of intravenous antibiotics he continued to have loose bloody stools, and on the third day after catheterization stool specimens were positive for rotavirus. On day 14 after the procedure, slow oral feedings were begun but the infant developed emesis and frequent loose stools that were guaiac positive. He underwent colonoscopy and barium enema that confirmed a descending and sigmoid colon stricture. Hemicolectomy and coloproctostomy were performed from which the infant recovered uneventfully.

\section{Discussion}

Infants with congenital heart disease may be at an increased risk for the development of necrotizing enterocolitis after cardiac catheterizations. ${ }^{5,6}$ Why this occurs has not been elucidated. In the previous studies the cases noted were uniquely clustered. The three cases of necrotizing enterocolitis reported by Cooke ${ }^{6}$ occurred after cardiac catheterization from the femoral vein. In Dickinson's study, ${ }^{5} 14$ episodes of necrotizing enterocolitis were observed following 111 cardiac catheterizations in neonates. The cases occurred during a 22-month period with two definite clusters. No discernible difference between those who developed necrotizing enterocolitis after catheterization versus those who did not was found. The cause for clustering was not determined although an infectious agent was suspected. In both of these prior studies the use of an ionic contrast agent for the cineangiograms was considered as a possible risk factor for the subsequent development of necrotizing enterocolitis. However, in the two infants presented in this report, necrotizing enterocolitis developed after the use of a nonionic contrast agent during cardiac catheterization. Ionic contrast material is therefore not a likely major confounding factor.

Necrotizing enterocolitis has been previously described after cardiac surgery. ${ }^{7}$ In addition, during the rotavirus epidemic in our hospital two infants developed necrotizing enterocolitis after cardiac surgery. These two cases occurred in a 3-month-old infant with tricuspid atresia after pulmonary artery banding and in a 4 -week-old infant after a Norwood procedure for hypoplastic left heart syndrome. Both infants had profuse diarrhea prior to the development of necrotizing enterocolitis. No other cases of enterocolitis occurred in any of the patients in the institution during this time period. Although rotavirus was not documented in the postsurgical cases it seems to be implicated as the causative agent. Cardiac surgery may also be a predisposing risk factor to necrotizing enterocolitis.

From our patients and previous studies it is clear that infants with congenital heart disease who develop necrotizing enterocolitis are those with symptomatic heart disease. This may be either cyanotic heart disease or acyanotic heart disease with congestive failure. Such infants may have reduced oxygen delivery to the gut. Reduced oxygen delivery could lead to gut ischemia and increased permeability of the intestinal lumen permitting the absorption of bacterial toxins. Yet not all infants with cyanotic heart disease or congestive heart failure develop necrotizing enterocolitis. Why an infant may develop necrotizing enterocolitis after rotaviral infection is not easily explained. Rotavirus is known to invade and replicate in the small bowel and cause epithelial cell destruction. ${ }^{8}$ The virus replication, however, is limited to the small bowel and does not occur in the colon. ${ }^{9}$ It is therefore difficult to implicate direct invasion of the colon by the virus as the cause of necrotizing enterocolitis. In the small intestine the destroyed epithelial cells are replaced by newly formed epithelial cells. The newly formed cells are deficient in disaccharidases and have abnormal glucose coupled sodium transportation that may be accompanied by malabsorption and metabolic acidosis. ${ }^{10}$ Alternatively, malabsorption in the small intestine may in turn lead to increased delivery of complex sugars and excess protein substrates to the colon. An alteration of the colonic flora may lead to the emergence of pathogenic bacteria, which may be an essential component in the development of necrotizing enterocolitis. Finally, damage to small bowel epithelium may permit absorption of bacterial endotoxins that act preferentially on colonic tissue.

An alternative explanation linking the physiology of the cardiac condition and the rotaviral infection is suggested by this study. During rotaviral infection significant diarrhea and dehy- 
dration may develop. The diarrhea leads to further dehydration in an infant whose feedings are held prior to the invasive procedure. The loss of intravascular volume could result in decreased blood flow to the gut, further reducing already marginal oxygen delivery. When the oxygen delivery is reduced below a critical level tissue hypoxia ensues and injury to the colon epithelium occurs, which may lead to the invasion of pathogenic organisms.

The four cases of enterocolitis described occurred in children with congenital heart disease during an epidemic of rotavirus infection at our institution. The presence of rotavirus is well documented in two cases. Previous studies showing an association between rotavirus and necrotizing enterocolitis $^{3,4}$ involved neonates located in a neonatal nursery. Relevant predisposing factors were: prematurity, respiratory distress syndrome, perinatal asphyxia, and umbilical vessel catheterization. The two infants presented herein were older ( 6 weeks and 6 months) and had none of the predisposing factors. To our knowledge these case reports represent the first documentation of older infants developing rotavirus-associated necrotizing enterocolitis.

\section{Implications}

These two cases suggest that there exists a relationship between rotavirus infection and the development of necrotizing enterocolitis in infants after cardiac catheterization. A prospective study designed to evaluate the role of rotaviral infection in the development of necrotizing enterocolitis after cardiac catheterization appears warranted. Meanwhile, pediatric cardiologists should proceed with caution when performing a cardiac catheterization during a known epidemic of rotavirus infection. During a rotaviral epidemic all infants should have rotaviral testing performed prior to cardiac catheterization. If testing is positive, the cardiac catheterization should be postponed until the infection has cleared. If the procedure cannot be postponed, the clinician must be aware of the signs and symptoms of necrotizing enterocolitis and its therapy.

\section{References}

1. Bell MJ, Ternberg JL, Feigin RD, et al. Neonatal necrotizing enterocolitis: Therapeutic decisions based on clinical staging. Ann Surg 1978; 187:1-7.

2. Rotbart HA, Levin MJ, Yolken RH, et al. An outbreak of rotavirus-associated neonatal necrotizing enterocolitis. J Pediatr 1983; 103:454-459.

3. Mogilner BM, Bar-Yochai A, Miskin A, et al. Necritizing enterocolitis associated with rotavirus infection. Isr J Med Sci 1983; 19:894-896.

4. Yolken RH, Kim HW, Clem T, et al. Enzyme-linked immunosorbent assay (ELISA) for detection of human reovirus-like agent of infantile gastroenteritis. Lancet 1977; 2:263-267.

5. Dickinson DF, Galloway RW, Wilkinson JL, et al. Necrotising enterocolitis after neonatal cardiac catheterization. Arch Dis Child 1982; 57:431-433.

6. Cooke RW, Meradji M, De Villeneuve VH. Necrotising enterocolitis after cardiac catheterization in infants. Arch Dis Child 1980; 55:66-68.

7. Kleinman $\mathbf{P}$, Winchester $\mathbf{P}$, Brill $\mathbf{P W}$. Necrotizing enterocolitis after open heart surgery employing hypothermia and cardiopulmonary bypass. Am J Roentgenol 1976; 127:757-760.

8. Mebus CA, Wyatt RG, Kapikian AZ. Pathology of diarrhea in gnotobiotic calves induced by human reoviruslike agent in infantile gastroenteritis. Vet Pathol 1977; 14:273-282.

9. Kapikian AZ, Kim HW, Wyatt RG, et al. Human reovirus-like agent as the major pathogen associated with "winter" gastroenteritis in hospitalized infants and young children. N Engl J Med 1976; 294:964-972.

10. Gall DG. Pathophysiology of viral diarrhea. In: Proceedings of 73rd Ross Conference on Pediatric Research: Etiology, pathology, and treatment of acute gastroenteritis. Ponte Verda Beach, Florida, March 20-22, 1977. 